\title{
Genetic influences on memory performance in familial Alzheimer disease
}

\author{
J.H. Lee, DrPH; A. Flaquer, MS; Y. Stern, PhD; B. Tycko, MD, PhD; and R. Mayeux, MD, MSc
}

\begin{abstract}
Objective: To investigate the heritability of memory and other cognitive measures in families with multiple individuals with Alzheimer disease $(\mathrm{AD})$ to determine if neuropsychological measures can be used to better understand genetic contributions to AD. Methods: The genetic contributions to the variation in declarative memory, attention, abstract reasoning, language, and visuospatial function using a variance component method were estimated. For memory scores, the proportion of genetic contribution was estimated, controlling for APOE. Results: The unadjusted heritability estimates for the declarative memory tasks ranged from 0.47 for delayed recall to 0.25 for delayed recognition, where a heritability estimate of 1 indicates that genetic factors explain all of the phenotypic variance and a heritability score of 0 indicates that genetic factors explain none. When adjusted for sex, age, education, and general intelligence, the heritability estimates increased to 0.60 for delayed recall and 0.41 for delayed recognition. None of the other cognitive tests showed heritability estimates as high as that observed for memory. When the influence of $A P O E$ was taken into account, the heritability estimates changed modestly for delayed recall and consistent long-term retrieval, whereas the estimates for other memory scores did not change, suggesting that APOE contributes little to these memory scores. Conclusions: Declarative memory in familial $\mathrm{AD}$ is under strong genetic influence, only part of which is attributable to $A P O E$. Memory performance should prove to be a useful phenotypic component in the investigation of the genetic basis of $\mathrm{AD}$.
\end{abstract}

NEUROLOGY 2004;62:414-421

Genetic investigation of Alzheimer disease $(\mathrm{AD})$ has relied on criteria-based diagnoses that include direct assessment of these cognitive functions, many of which are highly correlated. Memory loss, in particular, is a central feature of AD. Further, genetic influences on declarative or explicit memory have been established in studies of human twins, ${ }^{1-8}$ where the genetic component of verbal recall and recognition may be as high as 56\%. ${ }^{9}$ Remarkably, memory remains under substantial genetic influence into old age as observed in twins over age 75 even in the absence of dementia. ${ }^{4,5,10}$ To date, human studies are limited to twin studies.

The relation of the ApoE gene (APOE) with cognitive function has been investigated because of the strong association between the $\epsilon 4$ variant allele and $\mathrm{AD}$. In studies of healthy elderly populations, the $\epsilon 4$ allele appears to be associated with declining verbal memory but not with other cognitive functions. ${ }^{11-14}$

Given that memory decline precedes $\mathrm{AD}$ and that memory and $\mathrm{AD}$ have substantial genetic components, memory and other cognitive functions might be considered as endophenotypes for $\mathrm{AD}$. A better understanding of the genetic influences on memory or memory impairment and other cognitive functions affected in $\mathrm{AD}$ might clarify the relations between genetic factors that contribute to $\mathrm{AD}$. Here, we addressed two fundamental questions: 1) What is the underlying genetic contribution to various components of human memory in patients with $\mathrm{AD}$ and their family members, and (2) what is the remaining additive genetic contribution toward memory components after adjusting for the influence of $A P O E$ ? To address these two questions, we investigated the heritability of memory in a series of extended families with multiple individuals affected with $\mathrm{AD}$.

\begin{abstract}
Materials and methods. Subjects. We included data from 266 families that included 1,036 individuals participating in a study of familial $\mathrm{AD}$ in Caribbean Hispanic individuals. In brief, probands with $\mathrm{AD}$ were recruited from multiple sources, including clinics in the Dominican Republic and in Puerto Rico, the Alzheimer's Disease Research Center-Memory Disorders Center, and doctors' private offices in the Department of Neurology and the General Medical Services at Columbia University. Once a potential proband with $\mathrm{AD}$ was identified, the diagnosis was confirmed by standardized neurologic and neuropsychological evaluations. ${ }^{15}$ In addition, a structured family history interview was conducted with available family members to determine if the patient had living siblings or relatives with the disease. If the family history interview revealed additional affected family members, we interviewed and examined all other living relatives using the same battery of tests employed to assess the proband. The battery is described below. If more distant relatives of probands were af-
\end{abstract}

From the Gertrude H. Sergievsky Center (Drs. Lee, Stern, and Mayeux), Taub Institute for Research on Alzheimer's Disease and Aging Brain (Drs. Lee, Stern, Tycko, and Mayeux, A. Flaquer), and Departments of Neurology (Drs. Stern and Mayeux), Pathology (Dr. Tycko), and Psychiatry (Dr. Mayeux), College of Physicians and Surgeons, and Department of Epidemiology (Drs. Lee and Mayeux), School of Public Health, Columbia University, New York, NY. Supported by federal grants AG15473, AG08702, and AG07232; the Charles S. Robertson Memorial Gift for Alzheimer's Disease Research from the Banbury Fund; and the Blanchette Hooker Rockefeller Foundation.

Received March 31, 2003. Accepted in final form October 6, 2003.

Address correspondence and reprint requests to Dr. R. Mayeux, G.H. Sergievsky Center, Columbia University, 630 W. 168 St., New York, NY 10032; e-mail: rpm2@columbia.edu 
fected and agreed to participate, we ascertained those individuals as well as individuals linking the two branches of the pedigree.

The study was approved by the Institutional Review Board of the Columbia-Presbyterian Medical Center and the Bioethics National Committee for Research in the Dominican Republic. Informed consent was obtained from either the participant or the closest relative when the individual had dementia.

Neuropsychological tests. The same battery of neuropsychological tests was performed in all family members and probands in Spanish. The Spanish versions of these tests have been extensively tested and validated. ${ }^{16}$ The battery included assessments of memory, attention, abstract reasoning, language, and visuospatial function. Memory was tested using the Selective Reminding Test $(\mathrm{SRT})^{17}$ because of its predictive value for dementia. ${ }^{16}$ Specifically, six trials were given to learn a list of 12 unrelated words. After each attempt at recalling the list, the subject was reminded only of the words that were not recalled and then attempted to recall the entire list. Verbal memory was measured from the total recall score (maximum score $=72$ ) or the number of words recalled with and without reminding over the six trials (maximum score $=72$ ) Consistent long-term retrieval (CLTR) was measured by counting the number of items that were recalled consistently without an error (maximum score $=72$ ). Fifteen minutes after completing the SRT, delayed recall was measured by asking the subject to recall the original 12-word list (maximum score $=12$ ). Then delayed recognition was tested by asking for the words that were not recalled during the delayed recall test. Multiple-choice arrays were used for delayed recognition (maximum score $=12$ ). If all words were recalled, a perfect score of 12 was given. Nonverbal memory was examined using the Benton Visual Retention Test (BVRT). ${ }^{18}$ A recognition memory paradigm was used to assess nonverbal memory in a format that does not rely on constructional abilities. In this test, each of the 10 designs from the BVRT was shown for 10 seconds and then was removed. A four-item multiple-choice array was used to test recognition of each design (maximum score $=10$ ).

Additional neuropsychological tests were also included to assist in the diagnosis of dementia: orientation from the MiniMental State Examination, ${ }^{19}$ visuospatial ability from the Rosen Drawing Test ${ }^{20}$ and the matching subtest of the BVRT, ${ }^{18}$ the Boston Naming Test ${ }^{21}$ for naming, the Controlled Word Association Test $^{22}$ for verbal fluency, repetition and comprehension items from the Boston Diagnostic Aphasia Evaluation, ${ }^{23}$ abstract reasoning from the Similarities Subtest of the Wechsler Adult Intelligence Scale-Revised (WAIS-R), ${ }^{24}$ and the Identities and Oddities Subtest of the Mattis Dementia Rating Scale. ${ }^{25}$ This battery has been used in several studies of this Caribbean Hispanic population and has been previously described. ${ }^{26}$ For these neuropsychological tests, we computed correlation coefficients to determine pairwise relation between memory scores and other cognitive scores.

Statistical analysis. We estimated heritability using the variance component method as implemented by $\mathrm{SOLAR}^{27}$ to accommodate the complex pedigree structure of the participating families. This approach partitions the observed phenotypic variance into additive genetic variance and environmental variance. Heritability is expressed as a proportion of additive genetic variance over the total phenotypic variance and ranges from 0 to 1 , where 0 indicates no genetic influence and 1 indicates total genetic influence. We first applied a multivariate polygenic model adjusting for sex, age, and education to estimate heritability for memory and related cognitive traits. Then for the memory scores from the SRT, we refine the above model by adding overall intelligence, because overall intelligence-as estimated by age-scaled scores from the Similarities Subtest in the WAIS-R ${ }^{24}$-was reported to be correlated with both education and dementia. ${ }^{28}$ Further, to estimate the genetic contribution after controlling for the influence of $A P O E$, we included the number of $A P O E \in 4$ as a covariate while simultaneously applying the same multivariate polygenic model adjusting for sex, age, and education as above.

To further examine whether the estimated genetic influence applies to memory or is limited to $\mathrm{AD}$, we recomputed heritability using the same two models after coding the memory scores for the individuals with $\mathrm{AD}$ as unknown, thereby restricting the analysis to those without $\mathrm{AD}$ (this is shown in table 4).

Because the families were ascertained through probands with $\mathrm{AD}$ and because memory decline is the central manifestation of
$\mathrm{AD}$, we corrected for ascertainment bias by computing the likelihood after excluding probands. Moreover, because the families included multiple individuals with $\mathrm{AD}$, the distribution of memory scores based on these family members would be lower than that in the general population. This ascertainment bias could lead to reduced variability in neuropsychological scores among family members and would lead to an underestimation of heritability. Therefore, we constrained the mean and SD of the memory scores to those obtained from a population-based epidemiologic study of unrelated Caribbean Hispanic persons living in New York City. ${ }^{29}$ The epidemiologic study included data on a random sample of elderly over age 65 years, and approximately one-third were $\mathrm{Ca}$ ribbean Hispanics.

We compared the likelihood for a variance component model where the additive genetic variance for a quantitative trait locus $\left(\sigma^{2}{ }_{q}\right)$ was constrained to 0 vs the likelihood for a model where $\sigma^{2}{ }_{q}$ was estimated. We rejected the null hypothesis if the twice the difference in the log likelihood yielded a test statistic that is asymptotically distributed as a 50:50 mixture of a $\chi^{2}$ distribution with 1 degree of freedom and a point mass at $0 .{ }^{30}$ The test statistic assumed that the phenotypes of the family members follow a multivariate normal distribution; however, the variance component method has been shown to be robust to the violations of this assumption. ${ }^{27}$

APOE genotypes. Genotyping of $A P O E$ was performed using a modification of the published methods. ${ }^{31}$

Results. Subjects. The majority of the families were from the Dominican Republic, but approximately one-fifth of the families were recruited from Puerto Rico or elsewhere in the Caribbean. Table 1 shows the distribution of the 1,036 individuals from the 266 pedigrees. As shown in table 1 , this study included a large number of families with multiple individuals with $\mathrm{AD}$. Because the phenotype of interest is memory and related cognitive functions, we included the 28 families that lacked probands with probable or possible AD. However, these 28 families had 41 individuals with mild cognitive impairment. ${ }^{32}$ We obtained memory scores from all living family members when possible, including those who had $\mathrm{AD}$ as well as those who did not. Because of the nature of late-onset disease, the samples included probands and their siblings primarily, but other distant relationships such as aunts, uncles, and cousins of the probands were also included. The number of all possible pairwise relationships for family members who were examined is presented in table 1, illustrating the complexity of the pedigrees.

The proportion of women was higher than that of men (66 vs 34\%). Because our ascertainment strategy did not include age at onset as one of the eligibility criteria, the majority of the samples were of late-onset $\mathrm{AD}$; thus, we did not deliberately exclude early-onset $\mathrm{AD}$ families. The mean age of the individuals who were examined was 73.1 years (SD 3.2 years), and the average number of years of education was 6.2 years (SD 5.4 years). In this sample, approximately $50 \%$ were diagnosed as having AD. We screened for the presenilin (PS1) mutation in families where there was at least one individual with age at onset of $\mathrm{AD}$ younger than 55 years. We found 23 individuals from 19 families with this mutation and none with the amyloid- $\beta$ precursor protein $(A P P)$ mutation.

Correlations. To understand the relations among neuropsychological measures, we computed correlation coefficient (table 2). Correlation coefficients between memory and neuropsychological tests other than attention were high, with the median of 0.69 (range 0.50 to 0.87 ). However, for attention, the correlation coefficients were lower with a median of 0.36 (range 0.19 to 0.53 ). Performances 
No.

Distribution of families by no. of individuals with $\mathrm{AD}$

Families

individuals $\geq 5$ affecteds

3 affecteds

2 affecteds

1 affected

0 affected*

Total
4 affecteds

No. of pairwise relationships for individuals who have been examined $\dagger$

Parent-offspring

192

Siblings

1,572

Avunculars $\ddagger$

Half-siblings

First/second cousins

Half-avuncular

18
19
31
102
68
28
266

117

82

93

70

204

110

68

113

0

63

558

478

First cousins

Other relationships

Proportion of women, $\%$

$A P O E$ - 4 allele frequency, $\%$

28

* In the 28 families with no probable/possible Alzheimer disease (AD), there were 41 individuals with mild forms of dementia and 22 individuals with no sign of cognitive impairment.

$\dagger$ Numbers are not independent pairs.

$\ddagger$ Avunculars are typically siblings of the parents of the probands (e.g., aunts and uncles).

Table 2 Correlation coefficients between memory task scores and other cognitive test scores

\begin{tabular}{|c|c|c|c|c|c|c|c|c|c|c|c|}
\hline \multirow[b]{2}{*}{ Memory tasks } & \multicolumn{2}{|c|}{ Abstract reasoning* } & \multicolumn{2}{|c|}{ Attention* } & \multicolumn{5}{|c|}{ Language* } & \multicolumn{2}{|c|}{ Visuospatial* } \\
\hline & Similarities $\dagger$ & $\begin{array}{c}\text { Identities } \\
\text { and oddities } \dagger\end{array}$ & Shape $\dagger$ & $\mathrm{TMX}_{\dagger}^{\dagger}$ & Naming $\dagger$ & $\mathrm{CFL} \dagger$ & Animal $\dagger$ & Repetition $\dagger$ & Comprehension $\dagger$ & Rosen $\dagger$ & $\begin{array}{c}\text { Benton } \\
\text { Matching† }\end{array}$ \\
\hline \multicolumn{12}{|l|}{ SRT } \\
\hline Total recall & 0.68 & 0.79 & 0.42 & 0.46 & 0.82 & 0.75 & 0.86 & 0.78 & 0.77 & 0.69 & 0.80 \\
\hline Delayed recall & 0.64 & 0.63 & 0.29 & 0.32 & 0.66 & 0.66 & 0.69 & 0.59 & 0.64 & 0.59 & 0.70 \\
\hline $\begin{array}{l}\text { Delayed } \\
\quad \text { recognition }\end{array}$ & 0.61 & 0.83 & 0.51 & 0.54 & 0.85 & 0.71 & 0.76 & 0.81 & 0.79 & 0.70 & 0.80 \\
\hline CLTR & 0.61 & 0.54 & 0.19 & 0.27 & 0.57 & 0.63 & 0.61 & 0.50 & 0.58 & 0.53 & 0.63 \\
\hline BVRT & 0.71 & 0.73 & 0.37 & 0.44 & 0.77 & 0.77 & 0.73 & 0.70 & 0.74 & 0.75 & 0.87 \\
\hline
\end{tabular}

See Materials and Methods for a detailed description of each test.

* Areas of neuropsychological functions.

$\dagger$ Test items that were used to assess the cognitive functions listed above.

TMX = timed test to assess ability to differentiate letters; CFL = letter fluency; SRT = Selective Reminding Test; CLTR = consistent long-term retrieval; BVRT $=$ Benton Visual Retention Test. 
Table 3 Heritability coefficients for neuropsychological measures

\begin{tabular}{|c|c|c|c|}
\hline Neuropsychological test & Function & $H_{\text {adjusted }}^{2}(p$ value $)$ & $R^{* \dagger}$ \\
\hline Selective Reminding Test & Memory & & \\
\hline Total recall & & $0.32(<0.000001)$ & 0.12 \\
\hline Delayed recall & & $0.47(<0.000001)$ & 0.15 \\
\hline Delayed recognition & & $0.34(<0.000001)$ & 0.18 \\
\hline CLTR & & $0.40(<0.000001)$ & 0.08 \\
\hline Benton Recognition & & $0.25(<0.000001)$ & 0.22 \\
\hline MMSE, Orientation & & $0.25(<0.000001)$ & 0.19 \\
\hline WAIS, Similarities & Abstract reasoning & & \\
\hline Mattis Dementia Rating Scale & & $0.18(0.002106)$ & 0.14 \\
\hline Identities and Oddities & & $0.19(<0.000001)$ & 0.15 \\
\hline Shapes, timed & Attention & $0.20(0.000001)$ & 0.02 \\
\hline TMX, timed & & $0.30(<0.000001)$ & 0.03 \\
\hline Boston Naming Test, 15 words & Language & $0.19(<0.000001)$ & 0.29 \\
\hline Letter fluency (CFL) & & $0.31(<0.000001)$ & 0.20 \\
\hline Category fluency, animals & & $0.16(0.000010)$ & 0.20 \\
\hline BDAE, Repetition & & $0.17(<0.000001)$ & 0.27 \\
\hline BDAE, Comprehension & & $0.22(<0.000001)$ & 0.26 \\
\hline Rosen & Visuospatial & $0.24(0.000054)$ & 0.29 \\
\hline Benton Matching & & $0.32(<0.000001)$ & 0.22 \\
\hline
\end{tabular}

Refer to Materials and Methods for detailed descriptions.

* Adjusted for age, education, and sex.

$\dagger R^{2}$ represents the variance explained by covariates.

CLTR $=$ consistent long-term retrieval; MMSE = Mini-Mental State Examination; WAIS = Wechsler Adult Intelligence Scale; TMX = timed test to assess ability to differentiate letters; BDAE = Boston Diagnostic Aphasic Evaluation; Rosen = Rosen Drawing Test.

on tests of abstract reasoning, language, visuospatial function, and verbal fluency were very strongly related to memory performance. As expected, performance on all of the neuropsychological tests was significantly poorer in individuals with $\mathrm{AD}$ than in unaffected individuals. For example, the mean score for total recall was 9.4 for individuals with $\mathrm{AD}$ and 37.7 for unaffected individuals.

Heritability estimates based on all family members. When all family members were used in the analysis, heritability estimates were found to be moderate to high for nearly all of the memory tests (table 3). The unadjusted estimates (not presented in table 3) were 0.37 for total recall, 0.42 for delayed recall, 0.46 for delayed recognition, 0.38 for CLTR, and 0.41 for the BVRT. We conducted a subsequent analysis with covariates including age, sex, and education because of the known effects of age and education on neuropsychological test performance. Depending on the type of memory test performed, heritability remained high for most measures. For example, heritability for delayed recall (0.47) increased, whereas the nonverbal recognition test had a lower heritability estimate (0.25). The contribution of the covariates (i.e., environmental risk factors) also differed according to the type of memory test. The covariates explained a larger component of the phenotypic variance in the recognition test than in the long-term recall component of the SRT.

Heritability was also estimated for the other cognitive tasks, including abstract reasoning, attention, language, and visuospatial functions (see table 3). With few exceptions, heritability estimates were lower than those for the memory tasks. However, the heritability estimates for the letter fluency test and the Benton Matching Test were comparable with those for memory scores. As verbal fluency tasks often require generation of category exemplars that involve retrieval from semantic memory, ${ }^{33}$ heritability estimates in the range of those for memory might be expected.

We further refined the heritability model for memory scores by adding the Similarities Subtest of the WAIS-R ${ }^{24}$ as a means to estimate overall intelligence in addition to age, sex, and education (table 4). In this analysis, heritability estimates for memory scores increased for all, with the heritability estimates ranging from 0.60 for delayed recall to 0.38 for Benton recognition (see table 4). We then computed heritability estimates controlling for the influence of $A P O E$. With the $A P O E$ as a covariate, the estimate for residual genetic variance changed modestly for delayed recall and CLTR, whereas the estimates for genetic variance changed little for other memory scores, suggesting that the overall contribution by $A P O E$ toward memory scores is limited.

Heritability estimate of memory scores using unaffected family members. To determine the heritability of memory in the absence of $\mathrm{AD}$, we repeated the above analyses including only family members without $\mathrm{AD}$. The largest decrease in heritability estimate was observed for delayed 
Heritability estimate ( $p$ value)

\begin{tabular}{|c|c|c|}
\hline \multirow[b]{2}{*}{ Memory task } & \\
\hline & $\begin{array}{l}\text { Based on all family } \\
\text { members }\end{array}$ & $\begin{array}{c}\text { Based on individuals without } \\
\text { Alzheimer disease }\end{array}$ \\
\hline \multicolumn{3}{|l|}{ Total recall } \\
\hline Adjusted $H^{2}$ controlling for $A P O E \dagger$ & $0.41(<0.0000001)$ & $0.31(0.0001014)$ \\
\hline \multicolumn{3}{|l|}{ Delayed recall } \\
\hline Adjusted $H^{2}$ controlling for $A P O E \dagger$ & $0.65(<0.0000001)$ & $0.39(0.0004850)$ \\
\hline \multicolumn{3}{|l|}{ Delayed recognition } \\
\hline Adjusted overall $H^{2}$ & $0.41(<0.0000001)$ & $0.31(0.0000016)$ \\
\hline Adjusted $H^{2}$ controlling for $A P O E \dagger$ & $0.40(<0.0000001)$ & $0.31(0.0000016)$ \\
\hline \multicolumn{3}{|l|}{ CLTR } \\
\hline \multicolumn{3}{|l|}{ Benton recognition } \\
\hline Adjusted overall $H^{2}$ & $0.38(<0.0000001)$ & $0.32(0.0007329)$ \\
\hline Adjusted $H^{2}$ controlling for $A P O E \dagger$ & $0.34(0.0000003)$ & $0.32(0.0007329)$ \\
\hline
\end{tabular}

\footnotetext{
* We adjusted for age, sex, education, and Similarities of the Wechsler Adult Intelligence Scale-Revised as a measure of overall intelligence.

$\uparrow$ The heritability estimate for residual genetic variance, independent of $A P O E$, after adjusting for the same four covariates.
}

CLTR $=$ consistent long-term retrieval.

recall and CLTR. For other memory scores, the changes were relatively small. For delayed recall, heritability estimate for the model that included age, sex, education, and overall intelligence was lowered to 0.39 from 0.60 when individuals with $\mathrm{AD}$ were excluded. For CLTR, heritability estimate declined to 0.37 from 0.59 . On the other hand, the decrease in heritability estimate was relatively modest for other memory scores such as delayed recognition and Benton recognition. With the model that adjusted for $A P O E$, decline in heritability estimates for all memory scores was comparable with that for the estimates from the above model without $A P O E$.

Discussion. The overall genetic influence on verbal memory was high in families in which multiple persons had $\mathrm{AD}$, with a range of 0.30 to 0.65 , and it varied by the type of memory score used. In contrast, we found a relatively smaller degree of genetic influence on the other neuropsychological phenotypes including abstract reasoning, attention, language, and visuospatial functions. When $A P O E$ was controlled for, heritability estimates change modestly for delayed recall from 0.60 to 0.65 and for CLTR from 0.59 to 0.49 , but for other memory scores, heritability estimates changed little. Thus, whereas APOE appears to contribute to the memory phenotype to some extent in these families, other, as yet unidentified, genes also contribute substantially to memory. The degree of heritability remained high even when patients with $\mathrm{AD}$ in these families were removed from the analysis. Clearly, investigations to identify genes that underlie these memory scores are warranted, because such investigations could enhance our ability to localize genes that contribute to $\mathrm{AD}$ and its manifestations. Given the complexity of memory as a quantitative trait, however, it will be necessary to clarify the relations among components of memory and other cognitive functions affected in $\mathrm{AD}$ to optimize detection of the underlying genetic factors.

Several lines of evidence encourage further investigation of the genetic underpinning of memory in $\mathrm{AD}$ and in healthy individuals without dementia. Over the last decade, a number of scientists have established some of the molecular components of memory. The cyclic AMP-responsive element binding protein gene $(C R E B)$ played an important role in long-term potentiation, an analogue of long-term memory. ${ }^{34}$ With declarative memory, such as that examined in the tasks performed in this study, different stages may be encoded as changes in synaptic strength and correlate with the behavioral phases of short- and long-term memory. Short-term synaptic changes may involve simple modification of proteins, but long-term synaptic changes affect gene expression, new protein synthesis, and the formation of new connections..$^{35}$ Long-term memory in rodents relies on signaling pathways in protein kinase A, mitogen-activated protein kinase, and CREB-1, and the shift from short-term to long-term memory is also under molecular regulation. Thus, the molecular 
basis of memory has been established, but its genetic basis remains to be elucidated.

Human twins show that memory performance remains under strong genetic influence even with increasing age. In elderly Swedish twins, for example, the heritability of memory remained at $52 \%$ and that for general cognitive ability remains as high as $62 \%$ among elderly subjects..$^{4,5,10}$ However, other than twin studies that examine twins reared apart, twin studies cannot effectively separate shared environmental effects from shared genetic effects and thus overestimate genetic variance. Moreover, twins play a limited role in gene identification because only dizygotic twins provide information regarding allele sharing.

The results of our study extend the findings in twins regarding the heritability of memory to families with $\mathrm{AD}$. In the current study, we examine a simple model to measure additive genetic variance. Although the biological reality is far more complex and it may be useful to include more complex models (e.g., dominance, gene $\times$ gene interaction, and gene $\times$ environment interaction), it is important, first, to measure additive genetic variance for the purpose of gene identification as there is little power to detect more complex phenomena. Further, by examining the heritability of memory scores and their relation with one another, we can gain some insight into genetic contributions toward different cognitive domains as well as to AD. Even though we are limited in our ability to differentiate different forms of declarative memory because scores of different measures from the SRT were highly correlated, we did observe evidence for genetic heterogeneity in memory components and attempted to dissect different forms of declarative memory. Delayed recall and delayed recognition were highly correlated $(\rho=0.79$ ), yet the estimates of heritability were quite different (see table 4). The heritability estimate for delayed recall was 0.60 , and the estimate increased to 0.65 when $A P O E$ was taken into account. This is probably because both delayed recall and $A P O E$ are strong predictors of $\mathrm{AD}$. On the other hand, the heritability estimate for delayed recognition was lower than that for delayed recall ( 0.41 vs 0.60$)$, and the proportion of its additive genetic variance explained by $A P O E$ was negligible. It is of interest to note that the proportion of variance explained by known environmental risk factors such as age, sex, and education was small as well, indicating that we know very little about environmental risk factors for memory impairment. These observations suggest the following. Delayed recall tests measure memory storage and retrieval and are considered more difficult than recognition tests (e.g., delayed recognition or Benton recognition tests). Because of the difficulty of these tests, recall tests, compared with recognition tests, may require a greater degree of cognitive resources, some of which may be due to genetic factors..$^{36}$ This is further supported by the fact that with the addition of overall intelligence, the magnitude of the increase in heritability estimates was greater for these two recall tests. Taken together, it is plausible that these tests examined different cognitive functions, and the set of underlying genetic factors for the two memory tests might differ. Based on these observations, it may be possible to further explore the underlying factors for memory and $\mathrm{AD}$.

We cannot exclude the possibility that the genetic influences on memory reported here are restricted to familial AD. Here, we intentionally biased our sampling scheme for the linkage study by ascertaining multiple cases with $\mathrm{AD}$ to increase the likelihood of identifying $\mathrm{AD}$ susceptibility genes. Consequently, for the memory functions that are sharing the same causal pathway as $\mathrm{AD}$, the heritability estimates from the current study will be higher than those from a set of randomly selected families, irrespective of $\mathrm{AD}$ status in probands. This was supported by the facts that 1) the heritability estimates declined substantially for delayed recall and CLTR when AD individuals were excluded and 2) the heritability estimate was somewhat higher in families with three or more affected individuals compared with families with fewer affected individuals (0.49 vs 0.22$)$. However, there remained substantial additive genetic variance even when the individuals with $A D$ were removed. Similarly, the heritability remained unchanged when patients with dementia were excluded from their twin analysis. ${ }^{5}$ Because the battery of tests employed in the current study primarily evaluated explicit memory, additional work is needed to measure nondeclarative memory.

Our cohort differs from the US general population in a number of dimensions, and the generalizability of our study may be limited. One important dimension is socioeconomic level, including income and education. Under the regime of Trujillo from 1930 to 1961, public education in the Dominican Republic was limited, and the average years of education in our cohort is approximately 6 years. As education has been shown to be a protective factor, risk of dementia or memory impairment in this cohort may be higher than among the US population, where the average level of education is higher. In addition, most members of our cohort were of low economic level, and this may limit generalizability to the US general population. Genetically, Caribbean Hispanic persons constitute an admixed population of Spanish, African, and indigenous Tainos populations. ${ }^{37}$ Thus, the genetic background of this group differs from that of the US Caucasian majority and may differ from some Hispanic groups. However, the findings from this study are likely to be generalizable to the Hispanics in the USA. The majority of the Hispanic individuals are recent immigrants, with socioeconomic profiles that are similar to that of our cohort. Although these differences in socioeconomic status and genetic background in our cohort compared with the US general population might limit generalizability, certain fundamental biological processes may not be so different across ethnic groups. 
For example, the results from our gene localization study of $\mathrm{AD}$ on $12 \mathrm{p}$ showed remarkably similar peaks as those from the studies of the US white and European samples. ${ }^{38}$

It is well established that $\mathrm{AD}$ is a complex trait. In addition to the four genes that have been identified, several additional candidate chromosomal regions are under investigation. Yet the genes in these chromosomal regions remain difficult to identify using the current methods for a number of reasons. If these candidate regions truly harbor susceptibility genes, there will likely be several different genes with small effects. To enhance power to detect contributing genes with smaller effects as in most complex traits, it is useful to examine a quantitative endophenotype, an intermediate trait that is a component of the clinical outcome of interest. ${ }^{39,40}$ To study $\mathrm{AD}$ further, memory functions can be useful endophenotypes for $\mathrm{AD}$, as memory impairment is a central clinical characteristic of $\mathrm{AD}$. Given the ascertainment strategy of this study, this genetic study of memory will have greater power to detect genes that contribute to $\mathrm{AD}$ by searching for genes that contribute to memory impairment. Specifically, we can integrate the results from an $\mathrm{AD}$ genome-wide search with the results from a genome scan for memory scores. For example, if the $\mathrm{AD}$ and memory genome scans identify an approximately same candidate region, bivariate linkage analysis, which examines $\mathrm{AD}$ and memory simultaneously, can be performed to narrow down the candidate region. To identify genes that may contribute to both $\mathrm{AD}$ and memory functions, it would make sense to study the memory scores that showed a significant drop when individuals with $\mathrm{AD}$ were excluded in the analysis, as this is the phenotype that is likely to share the common pathway between memory impairment and $\mathrm{AD}$. On the other hand, if some chromosomal regions are associated with a memory phenotype alone, we can further explore the region using memory as the phenotypic endpoint. By comparing genetic factors that contribute to $\mathrm{AD}$, memory functions, or both, it will be possible to genetically dissect the pathways toward $\mathrm{AD}$ as well as memory functions unrelated to $\mathrm{AD}$.

We have demonstrated that the genetic basis of memory phenotype in these families with multiple $\mathrm{AD}$ patients is strong and that $A P O E$ plays a limited role. Because some memory systems have been mapped to certain regions of the brain, it may be possible to further narrow down the candidate genes by limiting candidate genes to those that are expressed in hippocampus and its related structures involving memory. Given that memory decline is a major manifestation of $\mathrm{AD}$, identifying genes related to memory traits may help us to understand the natural history of memory decline in $\mathrm{AD}$.

\section{Acknowledgment}

The authors thank the members of Estudio Familiar de Influencia Genetica en Alzheimer, the Sociedad Dominicana de Geriatria y Gerontologia, the Sociedad Dominicana de Neurologia y Neuro- cirugia, the Sociedad Dominicana de Psiquiatria, and the Associacion Dominicana Alzheimer y Similares, Inc., and the Bioethics National Committee for Research in the Dominican Republic.

\section{References}

1. Ando J, Ono Y, Wright MJ. Genetic structure of spatial and verbal working memory. Behav Genet 2001;31:615-624.

2. Carmelli D, Swan GE, DeCarli C, Reed T. Quantitative genetic modeling of regional brain volumes and cognitive performance in older male twins. Biol Psychol 2002;61:139-155.

3. Josselyn SA, Shi C, Carlezon WA Jr, et al. Long-term memory is facilitated by cAMP response element-binding protein overexpression in the amygdala. J Neurosci 2001;21:2404-2412.

4. McClearn GE, Johansson B, Berg S, et al. Substantial genetic influence on cognitive abilities in twins 80 or more years old. Science 1997;276 1560-1563.

5. McGue M, Christensen K. The heritability of cognitive functioning in very old adults: evidence from Danish twins aged 75 years and older. Psychol Aging 2001;16:272-280.

6. Reynolds CA, Finkel D, Gatz M, Pedersen NL. Sources of influence on rate of cognitive change over time in Swedish twins: an application of latent growth models. Exp Aging Res 2002;28:407-433.

7. Swan GE, Carmelli D. Evidence for genetic mediation of executive control: a study of aging male twins. J Gerontol 2002;57:P133-P143.

8. Wright M, De Geus E, Ando J, et al. Genetics of cognition: outline of a collaborative twin study. Twin Res 2001;4:48-56.

9. Swan GE, Reed T, Jack LM, et al. Differential genetic influence for components of memory in aging adult twins. Arch Neurol 1999;56: 1127-1132

10. Johansson B, Whitfield K, Pedersen NL, et al. Origins of individual differences in episodic memory in the oldest-old: a population-based study of identical and same-sex fraternal twins aged 80 and older. J Gerontol 1999;54:P173-P179.

11. Mayeux R, Small SA, Tang M, Tycko B, Stern Y. Memory performance in healthy elderly without Alzheimer's disease: effects of time and apolipoprotein-E. Neurobiol Aging 2001;22:683-689.

12. Haan MN, Shemanski L, Jagust WJ, Manolio TA, Kuller L. The role of APOE epsilon4 in modulating effects of other risk factors for cognitive decline in elderly persons. JAMA 1999;282:40-46

13. Raber J, Wong D, Yu GQ, et al. Apolipoprotein E and cognitive performance. Nature 2000;404:352-354.

14. Wilson RS, Schneider JA, Barnes LL, et al. The apolipoprotein E epsilon 4 allele and decline in different cognitive systems during a 6-year period. Arch Neurol 2002;59:1154-1160.

15. McKhann G, Drachman D, Folstein M, et al. Clinical diagnosis of Alzheimer's disease: report of the NINCDS-ADRDA Work Group under the auspices of Department of Health and Human Services Task Force on Alzheimer's Disease. Neurology 1984;34:939-944.

16. Jacobs DM, Sano M, Dooneief G, et al. Neuropsychological detection and characterization of preclinical Alzheimer's disease. Neurology 1995 45:957-962.

17. Buschke H, Fuld PA. Evaluating storage, retention, and retrieval in disordered memory and learning. Neurology 1974;24:1019-1025.

18. Benton AL. The Benton Visual Retention Test. New York: Psychological Corp., 1955.

19. Folstein MF, Folstein SE, McHugh PR. "Mini-Mental State." A practical method for grading the cognitive state of patients for the clinician. J Psychiatr Res 1975;12:189-198.

20. Rosen W. The Rosen Drawing Test. Bronx: Veterans Administration Medical Center, 1981.

21. Kaplan E, Goodglass H, Weintraub S. Boston Naming Test. Philadelphia: Lea \& Febiger, 1983.

22. Benton A, ed. FAS Test. Victoria, BC: University of Victoria, 1967.

23. Goodglass H, Kaplan E. The assessment of aphasia and related disorders. 2nd ed. Philadelphia: Lea \& Febiger, 1983

24. Wechsler D. WAIS-R manual. New York: Psychological Corp., 1981.

25. Mattis S. Mental status examination for organic mental syndrome in the elderly patient. In: Bellak L, Karasu T, eds. Geriatric psychiatry. New York: Grune and Stratton, 1976

26. Stricks L, Pittman J, Jacobs DM, Sano M, Stern Y. Normative data for a brief neuropsychological battery administered to English- and Spanish-speaking community-dwelling elders. J Int Neuropsychol Soc 1998;4:311-318.

27. Almasy L, Blangero J. Multipoint quantitative-trait linkage analysis in general pedigrees. Am J Hum Genet 1998;62:1198-1211.

28. Leibovici D, Ritchie K, Ledesert B, Touchon J. Does education level determine the course of cognitive decline? Age Ageing 1996;25:392-397.

29. Tang $M X$, Cross $P$, Andrews $H$, et al. Incidence of $A D$ in AfricanAmericans, Caribbean Hispanics, and Caucasians in northern Manhattan. Neurology 2001;56:49-56.

30. Self SG, Liang K-Y. Asymptotic properties of maximum likelihood estimators and likelihood ratio tests under the nonstandard conditions. J Am Stat Assoc 1987;82:605-610. 
31. Hixson JE, Vernier DT. Restriction isotyping of human apolipoprotein E by gene amplification and cleavage with HhaI. J Lipid Res 1990;31: 545-548.

32. Petersen RC, Smith GE, Waring SC, et al. Mild cognitive impairment: clinical characterization and outcome. Arch Neurol 1999;56:303-308.

33. Rosen VM, Engle RW. The role of working memory capacity in retrieval. J Exp Psychol Gen 1997;126:211-227.

34. Bartsch D, Casadio A, Karl KA, Serodio P, Kandel ER. CREB1 encodes a nuclear activator, a repressor, and a cytoplasmic modulator that form a regulatory unit critical for long-term facilitation. Cell 1998;95:211223 .

35. Kandel ER. The molecular biology of memory storage: a dialogue between genes and synapses. Science 2001;294:1030-1038.
36. Lee JH. Genetic evidence for cognitive reserve: variations in memory and related cognitive functions. J Clin Exp Neuropsychol 2003;25:594613

37. Moya Pons F. The Dominican Republic: a national history. New Rochelle: Hispaniola Books, 1995.

38. Mayeux R, Lee JH, Romas SN, et al. Chromosome-12 mapping of lateonset Alzheimer disease among Caribbean Hispanics. Am J Hum Genet 2002;70:237-243

39. Rice JP, Saccone NL, Rasmussen E. Definition of the phenotype. Adv Genet 2001;42:69-76.

40. Almasy L, Blangero J. Endophenotypes as quantitative risk factors for psychiatric disease: rationale and study design. Am J Med Genet 2001 105:42-44.

\section{MARK YOUR CALENDARS!}

AAN 56th Annual Meeting in San Francisco

April 24-May 1, 2004

Moscone Convention Center

San Francisco, CA

The 56th Annual Meeting Scientific Program highlights leading research on the most critical issues facing neurologists. More than 1,000 poster and platform presentations cover the spectrum of neurology-from updates on the latest diagnostic and treatment techniques to prevention and practice management strategies. 